\title{
A incidência de cesáreas e a remuneração da assistência ao parto
}

\author{
Juan Stuardo Yazlle Rocha* \\ Pabla Clotilde Ortiz* \\ Yang Tai Fung ${ }^{* *}$
}

Os autores estudam a incidência de cesáreas no total de partos ocorridos nos hospitais de Ribeirão Preto (SP), antes e depois da instituição da equiparação da remuneração ao parto por via baixa e por cesariana pela Previdência Social. Esta medida da Previdência Social não alterou a tendência à elevação da incidência de cesáreas no periodo do estudo (1978-1981). Observou-se a existência de um gradiente na taxa de cesáreas, sendo mais baixa a incidência média de cesáreas entre as pacientes não-pagantes e a mais elevada nas pacientes particulares, ficando a incidência de cesáreas entre as pacientes previdenciárias em uma posição intermediária. Atribuiu-se este gradiente a diferenças na modalidade de assistência, segundo a classe social das pacientes.

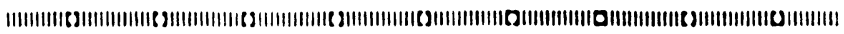

Diversos trabalhos têm demonstrado que nos últimos anos a incidência da cesárea vem aumentando, em diversas cidades, regiões e em diferentes países ${ }^{1,7}, 9,10$. Como razões que explicam esta elevação tem-se assinalado o alargamento das causas que levam à indicação de cesárea, classificadas como conquistas da obstetrícia atual, tais como os avanços no diagnóstico da anóxia crônica, da perinatologia e da diminuição dos riscos nas intervenções cirúrgicas ${ }^{1}$. Outras causas seriam o aumento da proporção de primíparas no total das parturientes, as cesáreas de repetição, as cesáreas a pedido (envolvendo a laqueação tubária) e, associadas às pacientes particulares, aponta-se a maior responsabilidade do médico e a procura de maior ganho (fator espúrio) $)^{1,5}$.

Em relação aos fatores de ordem técnica, trabalho de Marcoux \& Fabia, na região de Quebec (Canadá) ${ }^{9}$, mostra a elevação da incidência da cesárea entre 1973 e 1979 (de 7,9 a $13,6 \%$ ), assinalando que a cesárea de repetição foi responsável por $40 \%$ do aumento observado, sendo o restante
* Departamento de Medicina Social da Faculdade de Medicina de Ribeirão Preto USP

** Aluno da Fac. de Medicina de Ribeirão Preto - USP
Recebido para publicação em $10 / 07 / 85$ 
devido a problemas de distocia, má apresentação fetal. Gardonyi Carvalheiro5, em estudo realizado em Ribeirão Preto, referente aos anos de 1972 a 1974, constatou a elevação da incidência da cesárea nos partos das mulheres residentes no município (de 18,05 a 24,1\%). Constatou, também, aumento da incidência com a elevação do grupo etário das pacientes, desde $19,2 \%$, para o grupo de 15 a 19 anos, até $25,8 \%$, para o grupo de 40 anos. Entretanto, é a diferença da incidência de cesáreas entre classes sociais e/ou sistemas de financiamento da assistência ao parto que mais tem chamado a atenção de autores nacionais, provocando denúncias e até perplexidade ${ }^{1}$. Bastos ${ }^{11}$ encontrou no Rio de Janeiro (1970), em próprios da Previdência Social, incidências que variavam de 7,2 a $13 \%$, enquanto que na rede contratada elas ascendiam a mais de $30 \%$. Gardonyi Carvalheiro ${ }^{5}$ encontrou, entre as mulheres residentes no município de Ribeirão Preto, em 1974, incidência de $21,9 \%$ para as indigentes, $23,6 \%$ para as previdenciárias e $36,8 \%$ para as pacientes particulares. Camano \& Mattar $^{1}$ citam dados de diversos autores, referentes a São Paulo, Rio de Janeiro, Porto Alegre e Sul do Brasil, entre 1979 e 1982. Apesar da variação entre as faixas de incidência referidas, é constante o gradiente já mencionado antes; mais baixa incidência de cesáreas entre pacientes indigentes, intermediária entre pacientes de convênios previdenciários e mais altas entre pacientes particulares (entre 11 e $25 \%$ no caso das primeiras, de 18 a $40 \%$ no das segundas e 33 a $80 \%$ no das últimas).

Estes dados levam à convicção de estarmos diante de dois problemas distintos: o primeiro é a elevação da incidência da cesárea como resultado da evolução técnico-científica (alargamento das indicações, etc.) observado em diferentes países. Outro, é o responsável pelo diferencial de incidência de cesáreas entre classes sociais, não devidamente explicado e que tem sido relacionado, até agora, ao diferencial de remuneração entre os diferentes tipos de parto. Demonstrar esta relação foi o objetivo específico de um trabalho de Gentile de Mello ${ }^{6}$ no qual pretendeu mostrar em que medida, com que intensidade, desconsiderando as variáveis de ordem técnica, o regime de livre escolha e pagamento por unidade de serviço responde pelo incremento dos atos médicos e cirúrgicos. E, mais adiante: Como se não bastassem todos esses elementos de conviç̧ão sobre a influência negativa do regime de livre escolha e pagamento por unidade de serviço, vale rememorar a proposição do Conselho $R e$ gional de Medicina e da Associação Médica de Minas Gerais, apresentada ao Ministro do Trabalho e Previdência Social, em 1964, no sentido de "que o INPS pagasse um preço único por atendimento obstétrico, fosse ele parto normal, a fórceps, curetagem por aborto, parto duplo, cesárea mais 
histerectomia, etc. Uma oportunidade de testar o resultado da proposição acima veio com a publicação da Portaria MPAS/SSM-116, de 13 de dezembro de 1979, que estabeleceu a equiparação da remuneração médica nos casos de parto normal ou cesáreo.

Com base nestes fatos, resolvemos estudar a evolução das proporções de parto por via baixa e cesárea nos hospitais de Ribeirão Preto, durante os anos de 1978 a 1981, isto é, dois anos antes e depois da implantação da equiparação da remuneração ao parto pelo INAMPS.

\section{OBJETIVO}

Verificar se o estabelecimento de igual nível de remuneração ao médico na assistência ao parto por via baixa e cesárea, no sistema de assistência médica previdenciária, a partir de dezembro de 1979 , alterou a proporção de partos cinúrgicos em relação ao total, no município de Ribeirão Preto (SP), no período de 1978 a 1981.

\section{MATERIAL E MÉTODO}

A cidade de Ribeirão Preto é centro médico regional que conta com recursos médico-hospitalares diferenciados que atendem a totalidade da demanda local de hospitalizações ${ }^{12}$ e a grande demanda regional. No município, existem diversos sistemas de financiamento da assistência médico-hospitalar, desde o pagamento direto (Particulares), sistemas previdenciários oficiais (INAMPS, IAMSPE), sistemas de seguro-saúde privados (cooperativas médicas e empresas médicas) até o atendimento a não-pagantes (indigentes). $\mathrm{Na}$ cidade existe, também, um sistema de informações de dados de hospitalizações ${ }^{3}$, o Centro de Processamento de Dados Hospitalares (CPDH) do Departamento de Medicina Social da Faculdade de Medicina de Ribeirão Preto que centraliza dados da totalidade das hospitalizações no município, processando regularmente esses dados. Dos 13 hospitais existentes na cidade de Ribeirão Preto, cinco contavam, no período do estudo, com serviços permanentes de obstetrícia atendendo à quase totalidade dos partos que ocorrem no município. O hospital A é tradicional estabelecimento filantrópico que contava com um serviço de maternidade para a assistência a gestantes indigentes, o qual foi desativado ao final do primeiro ano do nosso estudo (1978), permanecendo o serviço para particulares e previdenciários. Os hospitais $\mathrm{B}$ e $\mathrm{C}$ atendem principalmente a previdenciários e segurados de sistemas privados de assistência. O hospital D atende 
quase exclusivamente a pacientes particulares e o hospital E é instituição acadêmica de ensino/investigação, com grande demanda regional, prestando assistência gratuita, uma parte da qual é dirigida a pacientes propriamente indigentes.

No presente estudo, partimos da constatação de que as parturientes se distribuem entre as categorias de internação (fontes de financiamento) na mesma proporção da totalidade dos pacientes de cada estabelecimento ${ }^{5}, 12$.

Os partos incluídos no estudo foram classificados em dois grandes grupos: parto por via baixa, incluindo os partos normais e os com complicações, e os partos cesareanos. Com esta finalidade, utilizaram-se as Listagens Trimestrais das hospitalizações e, quando necessário, os cartões perfurados das folhas de alta dos pacientes. Para a definição do tipo de parto, foram levados em conta o quarto e o quinto dígitos associados ao diagnóstico da causa da internação nos casos de parto, segundo a 8 a e 9a Revisões da Classificação Internacional de Doenças, observando a compatibilização da codificação utilizada nas mesmas.

\section{RESULTADOS E DISCUSSÃO}

$\mathrm{Na}$ tabela 1, é apresentada a distribuição do número médio de hospitalizações nos estabelecimentos incluídos no estudo, segundo categoria da internação, nos anos de 1978 a 1981. Vemos que, no hospital A, $68,4 \%$ das hospitalizações são financiadas pela Previdência Social, seguidas de outros seguros-saúde (oficiais e privados) (11,52\%), assistência a indigentes $(9,10 \%)$ e $6,87 \%$ de pacientes particulares. Este hospital desativou seu serviço para indigentes em fins de 1978; naquele ano, as internações desta categoria constituíaram $21,6 \%$ do total, enquanto que, no último ano do estudo, ficaram reduzidas a $1,5 \%$, apenas. Nos hospitais B e $\mathrm{C}$, predominam as internações previdenciárias, respectivamente 93 e $85 \%$ do total, sendo que, neste último, ainda houve um número significativo de pacientes particulares $(9,63 \%)$. O hospital D atende principalmente a pacientes particulares $(68,25 \%)$, sendo o restante financiado por seguros-saúde oficiais $(12,56 \%)$ e privados $(19,19 \%)$. O hospital $\mathrm{E}$ é instituição universitária que presta assistência gratuita a pacientes chamados indigentes, os quais constituíam $98,9 \%$ dos internados no período.

$\mathrm{Na}$ tabela 2, é apresentada a distribuição do total de partos realizados segundo a via de resolução, em cada um dos anos do estudo. No período de 1978 a 1981, foram realizados 36.956 partos, sendo 26.124 por via baixa e 10.832 por cesárea. O total anual de partos variou de 8.844 , em 1978, a 10.270, em 1981, e a proporção de partos cesarianos variou 
de $26,5 \%$ a $30,7 \%$, com proporção geral no período de $29,3 \%$. Estas proporções são semelhantes às referidas por Neiva ${ }^{10}$ para hospitais contratados pelo INAMPS, no mesmo período. É semelhante também à tendência geral crescente de partos cesarianos no total de partos, nos dados citados por Neiva e no presente estudo, apresentando, nos nossos dados, uma elevação de $15,8 \%$.

\section{TABELA 1}

Distribuiçāo do número médio de hospitalizaçōes nos estabelecimentos $A, B, C, D, E$, segundo a categoria da internação (Anos 1978 a 1981)

\begin{tabular}{|c|c|c|c|c|c|c|c|c|c|c|}
\hline \multirow{2}{*}{ Categoria } & \multicolumn{2}{|c|}{ A } & \multicolumn{2}{|c|}{ B } & \multicolumn{2}{|c|}{$\mathrm{C}$} & \multicolumn{2}{|c|}{$\mathrm{D}$} & \multicolumn{2}{|c|}{$E$} \\
\hline & No & $\%$ & No & $\%$ & No & $\%$ & No & $\%$ & Nọ & $\%$ \\
\hline PARTICULAR & 1.022 & 6,87 & 213 & 1,90 & 1.040 & 9,63 & 2.000 & 68,25 & 1.290 & 0,78 \\
\hline INDIGENTE & 1.359: & 9,10 & 58 & 0,50 & 19 & 0,20 & - & - & 16.655 & 98,90 \\
\hline INAMPS $^{1}$ & 10.181 & 68,40 & 10.543 & 93,00 & 9.178 & 85,00 & 1 & 0,03 & 17 & 0,10 \\
\hline SASSOM $^{2}$ & 240 & 1,61 & 183 & 1,60 & 52 & 0,50 & - & - & - & - \\
\hline IAMSPE $^{3}$ & 357 & 2,50 & 102 & 1,00 & 190 & 1,80 & 367 & 12,53 & - & - \\
\hline OUTROS & 1.718 & 11,52 & 218 & 2,00 & 318 & 2,87 & 562 & 19,19 & 25 & 0,20 \\
\hline TOTAL & 14.872 & 100,00 & 11.317 & 100,00 & 10.797 & 100,00 & 2.930 & 100,00 & 16.826 & 100,00 \\
\hline
\end{tabular}

* Serviço desativado a partir de 1979.

1. Instituto Nacional de Assistência Médica da Previdência Social

2. Serviço de Assistência Social dos Municipiários de Ribeirão Preto

3. Instituto de Assistência Médica dos Servidores do Estado de São Paulo.

\section{TABELA 2}

Distribuição do total de partos realizados, segundo tipo de resolução,em cada um dos anos do estudo. Ribeiräo Preto (SP) 178-1981

\begin{tabular}{|c|c|c|c|c|c|c|c|c|c|c|}
\hline \multirow{2}{*}{$\begin{array}{l}\text { Tipo de } \\
\text { Parto }\end{array}$} & \multicolumn{2}{|c|}{1978} & \multicolumn{2}{|c|}{1979} & \multicolumn{2}{|c|}{1980} & \multicolumn{2}{|c|}{1981} & \multicolumn{2}{|c|}{ Total } \\
\hline & Nọ & $\%$ & Nọ & $\%$ & Nọ & $\%$ & No & $\%$ & No & $\%$ \\
\hline VIA BAIXA & 6.500 & 73,5 & 6.461 & 70,8 & 6.041 & 69,2 & 7.122 & 69,3 & 26.124 & 70,7 \\
\hline CESĀREA & 2.344 & 26,5 & 2.653 & 29,2 & 2.687 & 30,8 & 3.148 & 30,7 & 10.832 & 29,3 \\
\hline TOTAL & 8.844 & 100,0 & 9.114 & 100,0 & 8.728 & 100,0 & 10.270 & 100,0 & 36.956 & 100,0 \\
\hline
\end{tabular}

Fonte: CPDH do Depto de Medicina Social da FMRP-USP

Na tabela 3, é apresentada a distribuição do total de partos realizados em cada um dos hospitais incluídos no estudo, segundo o tipo de resolução. Vemos que, dos 36.956 partos realizados no período, 12.455 , foram efetuados no hospital C, 9.821 no hospital E e 8.202, 5.140, 1.341 nos hospitais $A, B$ e $D$, respectivamente. A incidência de cesá- 
rea foi de $31,8,23,7,27,1,31,2$ e $54,3 \%$, respectivamente, nos hospitais C, E, A, B e D. A proporção de cesáreas mais baixa foi a observada no hospital E, apresentando oscilação no período, embora sem elevação da mesma; todos os outros hospitais apresentaram elevação na proporção das cesáreas no período do estudo. O hospital $A$ teve a segunda taxa mais baixa de cesáreas, e os hospitais $\mathrm{B}$ e $\mathrm{C}$ apresentaram proporções muito semelhantes, elevando a taxa das mesmas acima de $30 \%$ durante os anos do estudo. A incidência mais elevada das cesáreas ocorreu no hospital $\mathrm{D}$, onde variou de 47,1 a $58,5 \%$ no período do estudo. Estes dados são compatíveis com aqueles apresentados por Camano \& Mattar $^{1}$, indicando menor proporção de cesáreas em clínicas gratuitas e a maior de todas nas clínicas privadas, situando em proporção intermediária as clínicas de convênio com a Previdência Social. Nos nossos dados, o serviço gratuito é o hospital universitário, que presta assistência regional, o que poderia justificar um nível um pouco mais elevado do que o referido por Goffi ${ }^{7}$.

TABELA 3

Distribuição do total de partos realizados, segundo o tipo de resolução, em cada um dos hospitais incluidos no estudo. Ribeirão Preto (SP) 1978-1981.

Hospital

A

\begin{tabular}{|c|c|c|c|c|c|c|c|c|c|}
\hline \multirow{2}{*}{$\begin{array}{l}\text { Tipo de } \\
\text { Parto }\end{array}$} & \multirow[b]{2}{*}{ Ano } & & & & & & & & \\
\hline & & 1978 & 1979 & 1980 & 1981 & 1978 & 1979 & 1980 & 1981 \\
\hline \multirow{2}{*}{ VIA BAIXA } & Nọ & 2082 & 1476 & 1237 & 1186 & 795 & 971 & 878 & 891 \\
\hline & $\%$ & 75,7 & 73,3 & 70,8 & 70,1 & 73,0 & 68,0 & 69,0 & 66,0 \\
\hline \multirow{2}{*}{ CESÄREA } & No & 667 & 537 & 511 & 506 & 294 & 457 & 395 & 459 \\
\hline & $\%$ & 24,3 & 26,7 & 29,2 & 29,9 & 27,0 & 32,0 & 31,0 & 34,0 \\
\hline \multirow{2}{*}{ TOTAL } & Nọ & 2749 & 2013 & 1748 & 1692 & 1089 & 1428 & 1273 & 1350 \\
\hline & $\%$ & 100,0 & 100,0 & 100,0 & 100,0 & 100,0 & 100,0 & 100,0 & 100,0 \\
\hline
\end{tabular}

Fonte: CPDH do Depto de Medicina Social da F.M.R.P. - USP.

Os hospitais B e C internam pacientes, principalmente do sistema previdenciário, embora o $\mathrm{C}$ tenha certo número de particulares e seguros-saúde privados; apesar disto, a proporção de cesáreas foi um pouco inferior à referida por Goffi, porém situada, na média, acima de $30 \%$, no periodo.

$\mathrm{O}$ obje tivo maior do presente estudo é verificar se mudança introduzida pela Previdência Social, igualando a remuneração médica nos casos de parto via baixa e cesárea, levaria à mudança na incidência das cesáreas; com esta finalidade, pudemos estudar a incidência de cesáreas nos 2 anos antecedentes e subseqüentes à implantação daquela medida 
em hospitais com menor (A) ou maior predomínio de pacientes previdenciários $(\mathrm{B}, \mathrm{C})$, tendo como parâmentros de comparação um hospital de pacientes pagantes (D) e outro de não-pagantes $(E)$. Em relação à primeira parte, no estudo em cada estabelecimento, vemos que, nos anos subseqüentes à implantação de igual remuneração ao parto, a incidência da cesárea não diminuiu e nem se estabilizou, mas, ao contrário, manteve tendência à elevação da taxa de cesáreas em todos os hospitais, com exceção do hospital E (Hospital Universitário - gratuito). Embora curto o período de observação após a implementação da Portaria MPAS/SSM 116, podemos dizer que ela não teve nenhum efeito imediato na incidência de partos cesáreos, nem em hospitais com clientela predominante de previdenciários nem muito menos nos outros hospitais onde poderiam ter ocorrido mudanças, caso tivesse havido uma redistribuição da demanda de pacientes entre os estabelecimentos estudados, o que parece não ter acontecido. A assertiva de que a elevada taxa de cesáreas

\begin{tabular}{|c|c|c|c|c|c|c|c|c|c|c|c|}
\hline \multicolumn{4}{|c|}{$\mathrm{C}$} & \multicolumn{4}{|c|}{ D } & \multicolumn{4}{|c|}{ E } \\
\hline 1978 & 1979 & 1980 & 1981 & 1978 & 1979 & 1980 & 1981 & 1978 & 1979 & 1980 & 1981 \\
\hline 2001 & 2001 & 1929 & 2564 & 148 & 160 & 151 & 167 & 1475 & 1853 & 1846 & 2314 \\
\hline 66,0 & 70,01 & 6,59 & 66,4 & 52,9 & 48,9 & 45,5 & 41,5 & 76,9 & 74,4 & 75,4 & 78,1 \\
\hline 807 & 855 & 997 & 1299 & 132 & 167 & 181 & 235 & 444 & 637 & 603 & 649 \\
\hline 28,7 & 29,9 & 34,1 & 33,6 & 47,1 & 51,1 & 54,5 & 58,5 & 23,1 & 25,6 & 24,6 & 21,9 \\
\hline 2807 & 2856 & 2929 & 3863 & 280 & 327 & 332 & 402 & 1919 & 2490 & 2449 & 2963 \\
\hline 100,0 & 100,0 & 100,0 & 100,0 & 100,0 & 100,0 & 100,0 & 100,0 & 100,0 & 100,0 & 100,0 & 100,0 \\
\hline
\end{tabular}

tem como causa (direta e mecanicamente) o sistema de remuneração e o diferencial do pagamento por atos prestados, pressupõe uma concepção da assistência médica, onde o sistema de pagamento é a causa determinante (preponderante) na decisão das condutas médicas. Esta concepção não se sustenta diante dos nossos resultados. Ou seja, a elevação da incidência de cesáreas não tem como causa o diferencial de remuneração ao médico nos casos de parto via baixa e cesárea, visto que a equiparação da remuneração (no caso das pacientes previdenciárias) não alterou a tendência à elevação da taxa das cesáreas.

Caderno de Saúde Pública, R.J., 1(4):457-466, out/dez, 1985. 
De outro lado, é importante destacar o predomínio, em todo o período do estudo, do gradiente de cesáreas já referido anteriormente, com a incidência mais baixa (incidência média igual a $23,7 \%$ ) nas pacientes do hospital $E$, de nãopagantes; incidência média intermediária (de 31,2 a $31,8 \%$ ) nos hospitais de pacientes previdenciários, e a taxa mais alta $(54,3 \%)$ no hospital de pacientes pagantes. A determinação deste gradiente não está devidamente elucidada até agora. A concepção da assistência médica como prática eminentemente técnico-científica leva a procurar a proporção certa ou adequada de cesáreas no total de partos de uma população de referência. A existência de três (ou mais) taxas de cesáreas levantaria a suspeita de taxas anormais de partos operatórios, se não houver justificativas clínico-epidemiológicas para o gradiente observado. Não se considera que o gradiente é inversamente proporcional ao risco obstétrico de cada um dos grupos considerados (a incidência de cesáreas é menor no grupo de maior risco - as pacientes nãopagantes); ou, então, assume-se que o gradiente deve-se a fatores extratécnicos, no caso a remuneração ao médico pelo tipo de parto. Nossos dados indicam não haver esta relação direta, como foi acima explicado.

Entendemos que o gradiente tem determinação extratécnica, porém de outro tipo. A prática da assistência ao parto, como o trabalho médico em geral, determina-se socialmente, não sendo portanto uma prática técnica (neutra) exclusivamente $^{8}$. Os corpos sociais e o trabalho médico assumem características específicas quan to à definição do objeto de trabalho, objetivos, instrumentos e instrumentalização, etc, segundo a classe social a que aquele corpo pertence e o tipo de assistência médica que essa classe tenha conquistado; médico e paciente tềm-expectativas diversas quanto à conduta a seguir e o tratamento subseqüente, segundo a classe social de ambos; daí decorre a possibilidade social de assumir uma conduta expectante ou intervencionista (numa situação concreta dada), aceitando ou não o risco implícito em cada um dos casos. Os sistemas de financiamento da assistência médico-hospitalar (seguros saúde oficiais e privados, pagantes e não-pagantes) atendem a classes sociais específicas de forma predominante ${ }^{4}$; estas classes apresentam diferenciais de risco diante da doença e diferentes niveis de acesso aos recursos médico-assistenciais. Diante disto, a incidência maior ou menor de cesáreas em diferentes grupos de população não se explica por uma ação deliberada do médico no sentido de aumentar ou diminuir os atos cirúrgicos, senão que é muito mais o resultado de diferentes modalidades de trabalho médico geradas socialmente e que, independentemente da vontade dos profissionais da saúde, condicionam a sua atuação. A prevalência de diferentes taxas de cesáreas entre 
as classes sociais pode ser entendida como uma das características da medicina de classes vigentes entre nós.

Os antagonismos sociais gerados pelo processo de produção capitalista (produção coletiva e apropriação privada dos produtos) podem ser atenuados deslocando à esfera do consumo os conflitos originados na esfera da produção ${ }^{2}$; a assistência médica, na sociedade capitalista, restabelece a desigualdade social dos corpos através da medicina de classes.

\section{CONCLUSÕES}

Nos nossos dados, observou-se tendência à elevação da taxa de incidência de cesáreas nos anos do estudo, tendência que não se alterou após o estabelecimento da equiparação na remuneração ao médico no parto por via baixa e no parto cesareano, não podendo afirmar-se, portanto, que o aumento das taxas de cesáreas seja devido ao diferencial de remuneração ao parto. Foi constatado um gradiente de incidência de cesáreas que é inversamente proporcional ao risco obstétrico, sendo mais baixa nas pacientes não-pagantes e mais elevada nas pacientes particulares, ficando a incidência de cesáreas entre as pacientes previdenciárias em uma posição intermediária. Estas diferenças não podem ser atribuídas a uma ação deliberada do profissional, senão que são o produto das diferenças estabelecidas na assistência médica em distintas modalidades, segundo as classes sociais das pacientes.

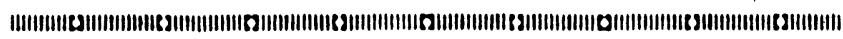

The authors studied the incidence of cesarean sections in deliveries in Ribeirão Preto (SP) hospitals before and after the implementation, by social security, of equal remuneration to the physician for surgical and non-surgical deliveries. Equal remuneration did not alter the increasing tendency of the incidence of cesarean sections in the years studied (1978-1981).

Differences were observed in cesarean ratios between non payer patients (lowest ratio) and particular patients (highest ratio), staying the patients of social security in an intermediate level. These differences must be due to different patterns of care give to the patients, according to their social class. 


\section{REFERENCIAS BIBLIOGRÁFICAS}

1. CAMANO, L. \& MATTAR, R. - Reflexões sobre a incidência da cesárea. R. Paul. Med. 101 (4): 160-4, 1983.

2. DONNANGELO, M.C.F. \& PEREIRA, L. - Saúde e sociedade. São Paulo, Duas Cidades, 1976.

3. FÃVERO, M.; YAZLLE ROCHA, J.S.; HADDAD, N. \& TERUEL, J.R. - Organização de um Centro de Informática Hospitalar em nível local. R. Paul. Hosp. 21 (4): 151-7, 1973.

4. FORSTER, A.C. - Hospitalizações e classes sociais. Ribeirão Preto, 1984. (Tese de Doutoramento, Depto de Medicina Social, Faculdade de Medicina de Ribeirão Preto-USP).

5. GARDONYI-CARVALHEIRO, C.D. - Padrões de atendimento ao parto no município de Ribeirão Preto, São Paulo, Brasil. Bol. Of. Sanit. Panam. 85 (3): 239-49, 1978.

6. GENTILE DE MELLO, C. - A epidemiologia da cesária. In: Saúde e assistência médica no Brasil. São Paulo, CEBES/ HUCITEC, 1977.

7. GOFFI, P.S. - Aumento da incidência da cesárea. Arq. Med. $A B C, 2: 5-7,1979$.

8. GONÇALVES, R.B.M. - Medicina e história, raízes sociais do trabalho médico. São Paulo, 1979. (Dissertação de Mestrado, Faculdade de Medicina-USP).

9. MARCOUX, S. \& FABIA, J. - Fréquence et indications des césariennes dans la région de Québec, Canada, en 1973 et 1979. Rev. Epidémiol. Santé Publique, 31 : 349-468, 1983.

10. NEIVA, J.G. - Indice de cesariana. Femina, 9:751, 1981. Apud CAMANO, L. \& MATTAR, R. - Reflexões sobre a incidência da cesárea. R. Paul Med. 101 (4): 160-4, 1983.

11. VIllela BASTOS, M. - Organização da assistência médica, uma contribuição para o estudo do problema brasileiro. Semana Médica, Lisboa, 1970. Apud GENTILE DE MELO, C. - A Epidemiologia da cesárea. In: Saúde e assisténcia médica no Brasil. São Paulo, CEBES/HUCITEC, 1977.

12. YAZLLE ROCHA, J.S. - Utilização de leitos hospitalares gerais em Ribeirão Preto. $R$. Saúde públ., 9:477-93, 1975. 